\title{
MicroRNA-139 inhibits the proliferation, migration and invasion of gastric cancer cells by directly targeting $\rho$-associated protein kinase 1
}

\author{
XUECHUN YU ${ }^{1 *}$, CHAOJIAN MA ${ }^{1 *}$, LING FU $^{1}$, JINGWU DONG $^{1}$ and JIE YING ${ }^{2}$ \\ Departments of ${ }^{1}$ Gastroenterology and ${ }^{2}$ Infectious Diseases, People's Hospital of Xuyi, Huai'an, Jiangsu 211700, P.R. China
}

Received March 17, 2016; Accepted October 24, 2017

DOI: $10.3892 / \mathrm{ol} .2018 .8038$

\begin{abstract}
The expression, function and underlying mechanisms of microRNA-139 (miR-139) in gastric cancer were investigated in the present study. Reverse transcription-quantitative polymerase chain reaction (RT-qPCR) was performed to detect miR-139 expression in gastric cancer tissues and cell lines. The effects of miR-139 overexpression on gastric cancer cell proliferation, migration and invasion were evaluated. $\rho$-associated protein kinase 1 (ROCK1) was predicted as a downstream target of miR-139 and its role in gastric cancer was assessed by bioinformatics analysis, luciferase reporter assay, RT-qPCR and western blot analysis. ROCK1 overexpression was established to investigate if the effects of miR-139 on gastric cancer cells may be attenuated. The results indicated that miR-139 was aberrantly downregulated in gastric cancer tissues and cell lines. Increased miR-139 expression reduced gastric cancer cell proliferation, migration and invasion. ROCK1 was demonstrated to be a direct target of miR-139 in gastric cancer and ROCK1 overexpression reversed the suppressive effects on gastric cancer cell proliferation, migration and invasion induced by miR-139 overexpression. The present study provides clear evidence demonstrating the anti-oncogenic activity of miR-139 in human gastric cancer, as mediated by the targeted downregulation of ROCK1.
\end{abstract}

\section{Introduction}

Gastric cancer is the fourth most common human cancer and the second most prevalent cause of cancer mortality worldwide (1). In total, $\sim 1,000,000$ cases of gastric cancer are newly diagnosed and $\sim 740,000$ mortalities occur every year (2).

Correspondence to: Dr Jie Ying, Department of Infectious Diseases, People's Hospital of Xuyi, 28 Hongwu Road, Xuyi, Huai'an, Jiangsu 211700, P.R. China

E-mail: yj_8611@126.com

*Contributed equally

Key words: microRNA-139, gastric cancer, proliferation, invasion, migration, $\rho$-associated protein kinase 1
Multiple risk factors have been identified to be involved in the initiation and progression of gastric cancer, including old age, smoking, alcohol consumption, obesity, unhealthy diet, low economic status, pernicious anemia, other chronic gastric diseases and Helicobacter pylori infection $(3,4)$. The current comprehensive care strategy for gastric cancer is a combination of surgery, chemotherapy and radiotherapy (5). However, $>50 \%$ of patients with advanced-stage gastric cancer succumb to recurrence or metastasis, despite undergoing curative gastrectomy (6). Gastric cancer typically has a 5-year overall survival rate of $\sim 15 \%$ and the median overall survival time for advanced-stage gastric cancer specifically is $<1$ year (7). Therefore, fully understanding the molecular mechanisms behind the progression of gastric cancer is imperative for the development of novel therapeutic strategies.

microRNAs (miRNAs/miRs) are endogenous, non-coding RNA molecules 21-23 nucleotides in length that can degrade mRNAs and suppress protein expression (8). miRNAs directly bind to the 3'-untranslated region (3'UTR) of their target mRNAs in a base pairing manner to induce mRNA degradation or suppress translation of the target proteins (9). miRNAs are involved in a variety of physiological and pathological processes, including cell proliferation, differentiation, apoptosis, invasion, migration and survival $(10,11)$. Differential expression of miRNAs between normal and tumor tissues has been reported in numerous human cancer types, suggesting a possible link between aberrant miRNA expression and the initiation and development of cancer (12). Furthermore, it has been demonstrated that miRNAs can function as oncogenes or tumor suppressors in gastric cancer and have significant regulatory functions in the disease. In a previous study, restored expression of the tumor suppressor miR-let-7a significantly inhibited gastric cancer cell growth, migration and invasion through the negative regulation of pyruvate kinase M2 (13). Furthermore, oncogenic miR-543 was demonstrated to promote the proliferation of gastric cancer via direct targeting of sirtuin 1 (14). Therefore, investigating the function of miRNAs in the tumor biology of gastric cancer may provide novel diagnostic and therapeutic targets for the treatment of this disease. In the present study, miR-139 expression in gastric cancer tissues and cell lines was detected and the effect of miR-139 overexpression on gastric cancer cell proliferation, migration and invasion was investigated. 


\section{Materials and methods}

Tissue specimens. The present study was approved by The Research Ethics Committee of The People's Hospital of Xuyi (Huai'an, China). A total of 30 patients (age range, 49-72 years; 19 males, 11 females) diagnosed with primary gastric cancer at The People's Hospital of Xuyi were included in the study between June 2014 and February 2016. Written informed consent was obtained from all patients. No participants had received chemotherapy or radiotherapy prior to surgery. Gastric cancer tissue and adjacent normal gastric tissue samples were collected, rapidly frozen in liquid nitrogen and kept at $-80^{\circ} \mathrm{C}$ until use.

Cell culture and oligonucleotide transfection. Human gastric cell lines (BGC-823, MGC-803 and SGC-7901) and the human immortalized gastric epithelial cell line (GES-1) were all purchased from American Type Culture Collection (Manassas, VA, USA). Cells were routinely cultured in RPMI 1640 medium (Gibco; Thermo Fisher Scientific, Inc., Waltham, MA, USA) with $10 \%$ fetal bovine serum (FBS; Gibco; Thermo Fisher Scientific, Inc.) in a humidified chamber with $5 \% \mathrm{CO}_{2}$ at $37^{\circ} \mathrm{C}$.

miR-139 mimic, miR-139 mimic negative control (NC), ROCK1 expression vector (pcDNA3.1-ROCK1) and control expression vector (pcDNA3.1-Control) were chemically synthesized by Shanghai GenePharma Co., Ltd. (Shanghai, China). The miR-139 mimics sequence was 5'-UCUACAGUG CACGUGUCUCCAGU-3' and the NC sequence was 5'-UUC UCCGAACGUGUCACGUTT-3'.

Gastric cancer cells were transfected with miRNA mimics $(100 \mathrm{pmol})$ or vector $(2 \mu \mathrm{g})$ with Lipofectamine ${ }^{\circledR}$ 2000 (Invitrogen; Thermo Fisher Scientific, Inc.). Following transfection (48 h), RT-qPCR was performed to determine miR-139 or ROCK1 mRNA expression. Cell proliferation, migration and invasion assays were conducted at 24 and $48 \mathrm{~h}$ post-transfection, respectively. Western blot analysis was carried out at $72 \mathrm{~h}$ following transfection.

Reverse transcription-quantitative polymerase chain reaction $(R T-q P C R)$. Total RNA was isolated from tissues or cell lines using TRIzol reagent (Invitrogen; Thermo Fisher Scientific, Inc.) according to the manufacturer's instructions. The relative expression of miR-139 was determined by RT-qPCR using the TaqMan Universal Master Mix (Applied Biosystems; Thermo Fisher Scientific, Inc.). The thermocycling conditions were as follows: $50^{\circ} \mathrm{C}$ for $2 \mathrm{~min}, 95^{\circ} \mathrm{C}$ for $10 \mathrm{~min}$; 40 cycles of denaturation at $95^{\circ} \mathrm{C}$ for $15 \mathrm{sec}$; annealing/extension at $60^{\circ} \mathrm{C}$ for $60 \mathrm{sec}$. RNU6b was used to normalize miR-139 expression. For ROCK1 mRNA expression, reverse transcription was performed using the RevertAid ${ }^{\mathrm{TM}}$ H Minus First Strand cDNA Synthesis kit (Thermo Fisher Scientific, Inc.), followed by PCR using SYBR Green Premix Ex Taq (Takara Biotechnology Co., Ltd., Dalian, China). The amplification was performed with cycling conditions as follows: $5 \mathrm{~min}$ at $95^{\circ} \mathrm{C}$, followed by 40 cycles of $95^{\circ} \mathrm{C}$ for $30 \mathrm{sec}$ and $65^{\circ} \mathrm{C}$ for $45 \mathrm{sec}$. $\beta$-actin was used as an endogenous control for ROCK1 mRNA expression. The primers were designed as follows: miR-139 forward, 5'-CAGCGGTCTACAGTGCACGT-3' and reverse, 5'-CAG TGCAGGGTCCGAGGT-3' U6 forward, 5'-GCTTCGGCA
GCACATATACTAAAAT-3' and reverse, 5'-CGCTTCACG AATTTGCGTGTCAT-3' ROCK1 forward, 5'-AGGAAGGCG GACATATTGATCCCT-3' and reverse, 5'-AGACGATAGTTG GGTCCCGGC-3' and $\beta$-actin forward, 5'-CCTGGCACC CAGCACAAT-3' and reverse, 5'-GCTGATCCACATCTG CTGGAA-3'. The relative expression of miR-139 and ROCK1 mRNA was analyzed using the $2^{-\Delta \Delta C q}$ method (15).

Cell proliferation assay. Cell proliferation was determined by MTT assay. Transfected cells were collected and seeded into 96-well plates at a density of $5 \times 10^{3}$ cells/well with $200 \mu \mathrm{l}$ RPMI 1640 medium (Gibco; Thermo Fisher Scientific, Inc.). Following incubation at $37^{\circ} \mathrm{C}$ for $24-96 \mathrm{~h}$, cells were incubated for $4 \mathrm{~h}$ at $37^{\circ} \mathrm{C}$ with $20 \mu \mathrm{l}$ MTT solution $(5 \mathrm{mg} / \mathrm{ml}$; Sigma-Aldrich; Merck KGaA). Dimethyl sulfoxide (150 $\mu$ l) was subsequently added to dissolve the crystals for $20 \mathrm{~min}$ at room temperature. The absorbance was measured at $490 \mathrm{~nm}$ with a microplate reader (Bio-Rad Laboratories, Inc., Hercules, CA, USA).

Cell migration and invasion assay. For the cell invasion and migration assays, Transwell plates (pore size, $8 \mu \mathrm{m}$ ) with and without a Matrigel coating were respectively used (BD Biosciences, Franklin Lakes, NJ, USA). A total of $1 \times 10^{5}$ transfected cells in $100 \mu 1$ FBS-free RPMI 1640 medium (Gibco; Thermo Fisher Scientific, Inc.) were added into the upper chamber, and the lower chamber was filled with $600 \mu \mathrm{l}$ RMPI 1640 medium (Gibco; Thermo Fisher Scientific, Inc.) containing 20\% FBS (Gibco; Thermo Fisher Scientific, Inc.). After incubation for $48 \mathrm{~h}$, cells on the upper surface of Transwell plates were removed with a cotton-tipped swab and cells migrating to the bottom of the filter were fixed with $100 \%$ methanol at room temperature for $15 \mathrm{~min}$ and stained with $0.1 \%$ crystal violet at room temperature for $15 \mathrm{~min}$. The migrated or invaded cells were counted in five fields using a light microscope (magnification, x100; BX51; Olympus Corporation, Tokyo, Japan).

Bioinformatics analysis. TargetScan (http://www.targetscan .org/) and PicTar (http://www.pictar.org/) were used to predict the target genes of miR-139.

Luciferase reporter assay. pGL3-ROCK1-3'UTR-wild-type (Wt) or pGL3-ROCK1-3'UTR-Mutant (Mut) luciferase reporter vectors (Shanghai GenePharma Co., Ltd.), along with miR-139 mimics or NC, were transfected into gastric cancer cells using Lipofectamine ${ }^{\circledR} 2000$ (Invitrogen; Thermo Fisher Scientific, Inc.). At $48 \mathrm{~h}$ post-transfection, the cells were assayed for luciferase activity using the Dual-Luciferase Reporter assay system (Promega Corporation, Madison, WI, USA) according to the manufacturer's instructions. The firefly luciferase activity was normalized to Renilla luciferase activity.

Western blot analysis. At $78 \mathrm{~h}$ post-transfection, total protein was isolated from transfected cells using radioimmunoprecipitation assay lysis buffer (1\% 4-nonylphenyl poly (ethylene glycol), $0.1 \%$ SDS, $100 \mu \mathrm{g} / \mathrm{ml}$ phenylmethylsulfonyl fluoride and $0.5 \%$ sodium deoxycholate in PBS). Protein concentration was determined using a bicinchoninic acid assay kit (Beyotime Institute of Biotechnology, Haimen, China). Protein 
$(20 \mu \mathrm{g})$ was separated using SDS-PAGE (10\% gel) and transferred to polyvinylidene fluoride membranes (Merck KGaA). Membranes were blocked with 5\% skimmed milk in TBS/0.1\% Tween for $1 \mathrm{~h}$ and subsequently incubated at $4^{\circ} \mathrm{C}$ overnight with primary antibodies against ROCK1 (1:1,000 dilution; catalog no. sc-17794; Santa Cruz Biotechnology, Inc., Dallas, TX, USA) or GADPH (1:1,000 dilution; catalog no. sc-47778; Santa Cruz Biotechnology, Inc.). Membranes were then incubated with goat anti-mouse horseradish peroxidase-conjugated secondary antibody (1:10,000 dilution; catalog no. sc-2005; Santa Cruz Biotechnology, Inc.). Protein bands were visualized using an enhanced chemiluminescence kit (Thermo Fisher Scientific, Inc.) and quantified with LabWorks Image Acquisition and Analysis Software (version 3; UVP, LLC, Phoenix, AZ, USA).

Statistical analysis. Statistical analysis was performed using SPSS 15.0 (SPSS Inc., Chicago, IL, USA). Data is presented as the mean \pm standard deviation. Statistical significance was determined using Student's t-test or one-way analysis of variance followed by the Student-Newman-Keuls test. $\mathrm{P}<0.05$ was considered to indicate a statistically significant difference.

\section{Results}

miR-139 expression is downregulated in human gastric cancer cell lines and tissues. miR-139 expression levels in gastric cancer and adjacent normal gastric tissues were detected by RT-qPCR. miR-139 expression was significantly downregulated in gastric cancer tissues compared with their matched adjacent normal gastric tissues (Fig. 1A; P<0.05). miR-139 expression levels were subsequently determined in BGC-823, MGC-803, SGC-7901 and GES-1 cell lines. Significantly reduced expression levels were observed in the gastric cancer cell lines compared with that in the GES-1 cell line, indicating that miR-139 expression may be downregulated in gastric cancer (Fig. 1B; P<0.05). BGC-823 and SGC-7901 cell lines exhibited relatively lower miR-139 expression among these three cell lines, and therefore were selected for subsequent experiments.

ROCK1 is a direct target of miR-139. Bioinformatics analysis was performed to further understand the mechanisms underlying the role of miR-139 in gastric cancer. The 3'UTR of ROCK1 was confirmed to contain the binding site of miR-139 at positions 538-545 (Fig. 2A). A luciferase reporter assay was performed to validate whether ROCK1 was a direct target of miR-139. Results revealed that miR-139 significantly reduced the luciferase activity in the pGL3-ROCK1-3'UTR-Wt compared with the NC (Fig. 2B; P<0.05), whereas pGL3-ROCK1-3'UTR-Mut activity was not significantly different. RT-qPCR and western blot analysis revealed that restored miR-139 expression significantly decreased ROCK1 mRNA (Fig. 2C; P $<0.05$ ) and protein (Fig. 2D; P 20.05 ) expression in BGC-823 and SGC-7901 cell lines. Only the BGC-823 and SGC-7901 cell lines were used as these exhibited relatively lower miR-139 expression among the three cell lines and were therefore selected for subsequent experiments. These results indicated that miR-139 may regulate ROCK1 expression in gastric cancer through interaction with the 3'UTR of ROCK1 mRNA.
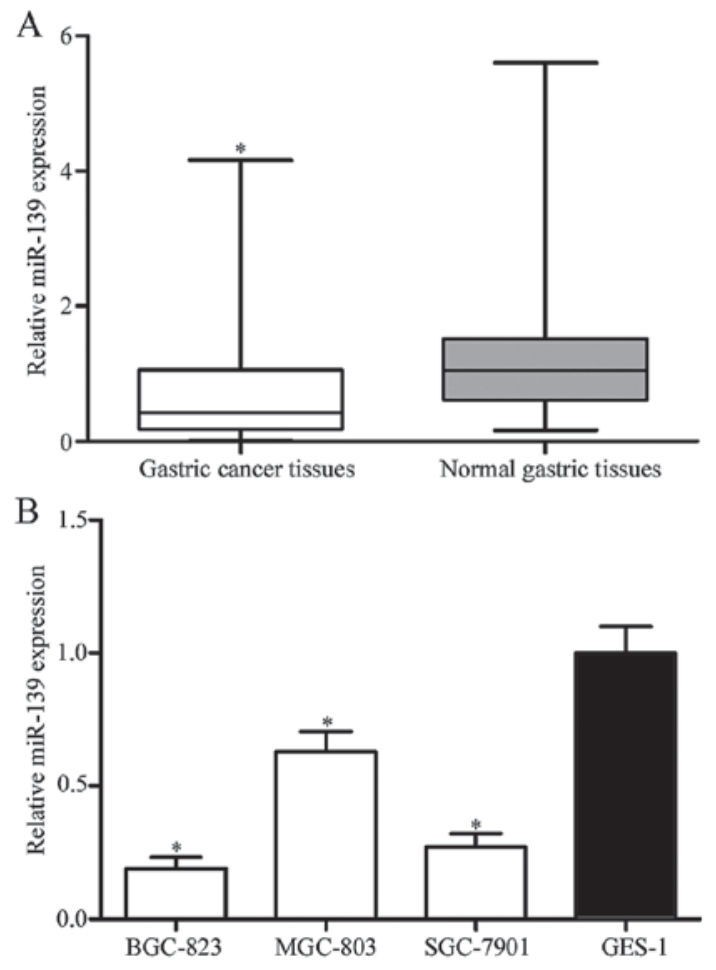

Figure 1. Downregulation of miR-139 expression in gastric cancer. (A) Reverse transcription-quantitative polymerase chain reaction results of miR-139 expression in primary gastric cancer and adjacent normal gastric tissues. (B) miR-139 expression levels in gastric cancer cell lines and a normal gastric cell line. ${ }^{*} \mathrm{P}<0.05$ vs. control. miR-139, microRNA-139.

miR-139 inhibits gastric cancer cell proliferation, migration and invasion by repressing ROCK1 expression. The downregulation of miR-139 observed in gastric cancer cell lines and tissues suggested that it may physiologically act as a tumor suppressor. Therefore, miR-139 mimics were transfected into SGC-7901 and BGC-823 cell lines. Significantly increased miR-139 expression compared with the NC was confirmed by RT-qPCR after $24 \mathrm{~h}$ (Fig. 3A; P<0.05). The effect of miR-139 expression on gastric cancer proliferation was investigated by MTT assay. Results demonstrated that BGC- 823 and SGC-7901 cell lines transfected with miR-139 mimics had significantly reduced cell proliferation at 72 and $96 \mathrm{~h}$ compared with the NC group (Fig. 3B; $\mathrm{P}<0.05$ ). Cell migration and invasion assays indicated that restored miR-139 expression significantly decreased the migration (Fig. 3C; $\mathrm{P}<0.05$ ) and invasion (Fig. 3D; P<0.05) abilities of the BGC-823 and SGC-7901 cell lines. Taken together, these results suggested that miR-139 acts as a tumor suppressor by inhibiting cell proliferation, migration and invasion.

The present study also investigated whether increased ROCK1 expression was able to attenuate the antitumor activity of miR-139 in gastric cancer cell lines. ROCK1 overexpression was established by transfection and confirmed by RT-qPCR (Fig. 3E; P<0.05). Subsequent assays demonstrated that ROCK1 overexpression reversed the suppressive effects induced by miR-139 on gastric cancer cell proliferation (Fig. 3B; $\mathrm{P}<0.05$ ), migration (Fig. $3 \mathrm{C} ; \mathrm{P}<0.05$ ) and invasion (Fig. 3D; P<0.05). These results indicated that miR-139 may inhibit gastric cancer proliferation, migration and invasion partially through the downregulation of ROCK1. 


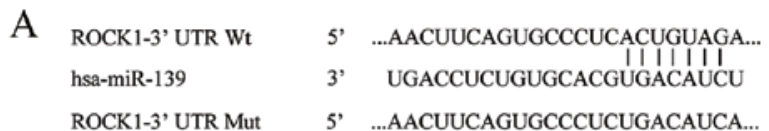

$\mathrm{B}{ }_{1.5} \ln _{\mathrm{NC}}$
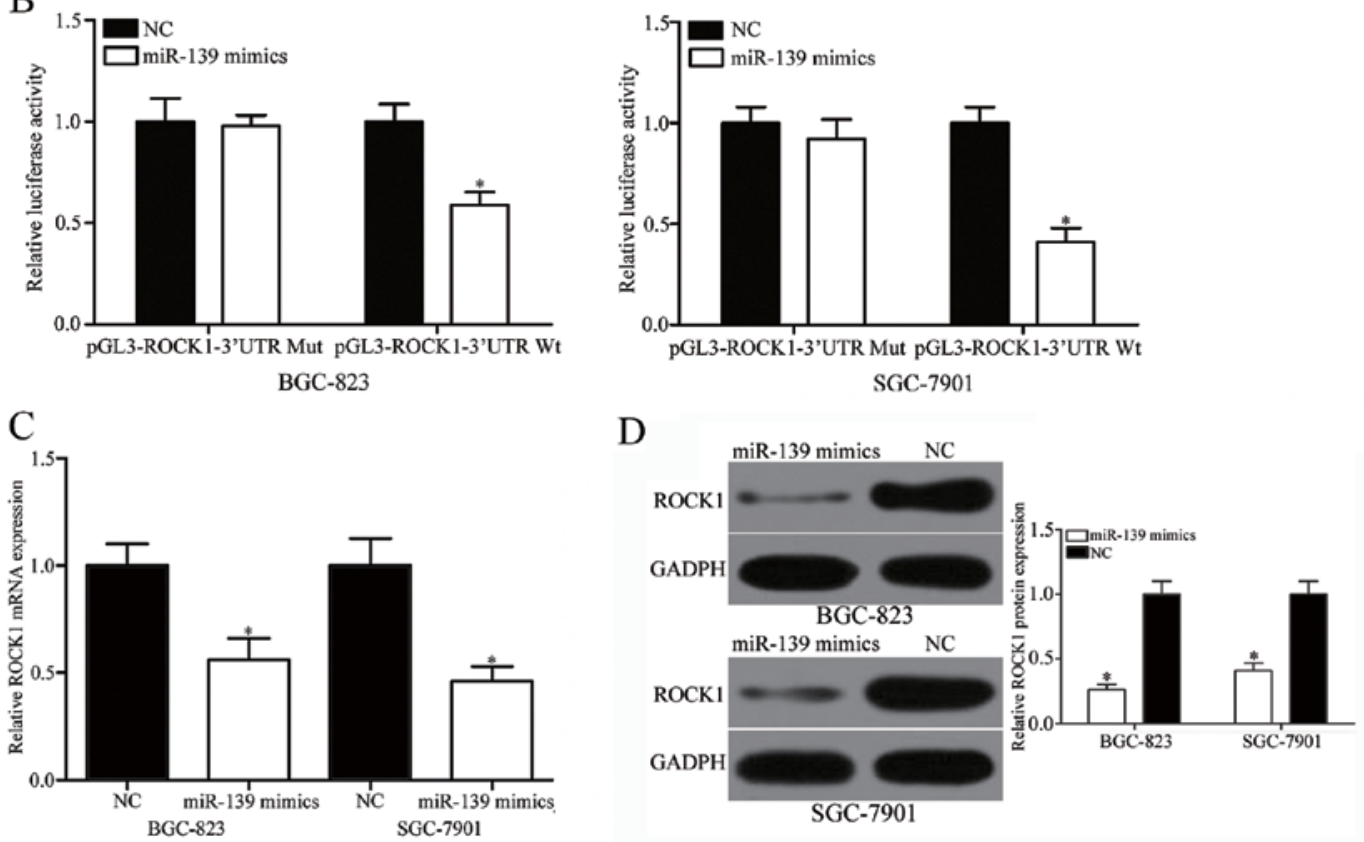

Figure 2. ROCK1 was confirmed as a target of miR-139. (A) Predicted consequential pairing of miR-139 and the target 3'UTR region of ROCK1 (Wt and Mut). (B) BGC-823 and SGC-7901 cells were co-transfected with pGL3-ROCK1-3'UTR-Wt or pGL3-ROCK1-3'UTR-Mut and miR-139 mimic or NC. (C) Reverse transcription-quantitative polymerase chain reaction detection of ROCK1 mRNA expression in BGC-823 and SGC-7901 cells transfected with miR-139 mimic or NC. (D) ROCK1 protein expression was detected by western blot analysis in BGC-823 and SGC-7901 cells transfected with miR-139 mimic or NC. "P<0.05 vs. control. miR-139, microRNA-139; ROCK1, $\rho$-associated protein kinase 1; hsa, Homo sapiens; Wt, wild-type; Mut, mutant; NC, negative control; UTR, untranslated region.

\section{Discussion}

To the best of our knowledge, the present study provides novel evidence of the downregulation of miR-139 in gastric cancer tissues and cell lines compared with adjacent normal gastric tissues and the normal gastric epithelial GES-1 cell line, respectively. The aim of the present study was to elucidate the biological function of miR-139 in gastric cancer carcinogenesis and progression. Restored miR-139 expression significantly inhibited gastric cancer proliferation, migration and invasion. Therefore, miR-139 may function as a tumor suppressor in gastric cancer.

Recent studies have revealed that low miR-139 expression occurs in various types of human cancer, including glioma (16), tongue squamous cell carcinoma (17), breast cancer (18), non-small cell lung cancer (19) and colon cancer (20). Furthermore, miR-139 expression has been demonstrated to associate with clinicopathological factors. Wong et al (21) reported that low miR-139 expression in hepatocellular carcinoma was significantly associated with poor patient prognosis and metastatic tumor characteristics, including venous invasion, microsatellite formation, an absence of tumor encapsulation and reduced differentiation. Shen et al (22) demonstrated that miR-139 expression was also downregulated in colorectal cancer tissues compared with that in adjacent non-tumorous tissues, and decreased miR-139 expression has also been associated with colorectal cancer progression and metastasis. Furthermore, in a study by Liu et al (23), miR-139 expression was demonstrated to be reduced in esophageal squamous cell carcinoma tissues, and this reduction was associated with lymph node metastasis. These findings indicate that increasing miR-139 expression may be useful in the prevention of cancer progression.

Several lines of evidences have demonstrated the role of miR-139 in a range of physiological and pathological processes. Wang et al (15) and Li et al (24) reported that miR-139 may inhibit glioma cell proliferation and invasion in vitro and in vivo. It has also been reported that miR-139 can suppress glioblastoma cell proliferation, migration and invasion, and induce apoptosis $(25,26)$. In non-small cell lung cancer, miR-139 decreases the proliferation and invasion of cancer cells (18). Additionally, miR-139 suppresses epithelial-mesenchymal transition, migration and invasion in hepatocellular carcinoma, and the incidence and severity of pulmonary metastasis in orthotopic liver tumors in mice is also reduced $(20,27)$. Furthermore, Zhang et al (28) revealed that colorectal cancer cell viability, colony formation, nude mice tumor growth, cell migration and invasion are repressed by miR-139, and Luo et al (29) demonstrated that proliferation, invasion and metastasis of laryngeal squamous carcinoma cells are also reduced. miR-139 functions as a tumor suppressor in esophageal squamous cell carcinoma by cell proliferation, migration and invasion inhibition, apoptosis induction and G0/G1 phase cell cycle arrest (22). These 
A
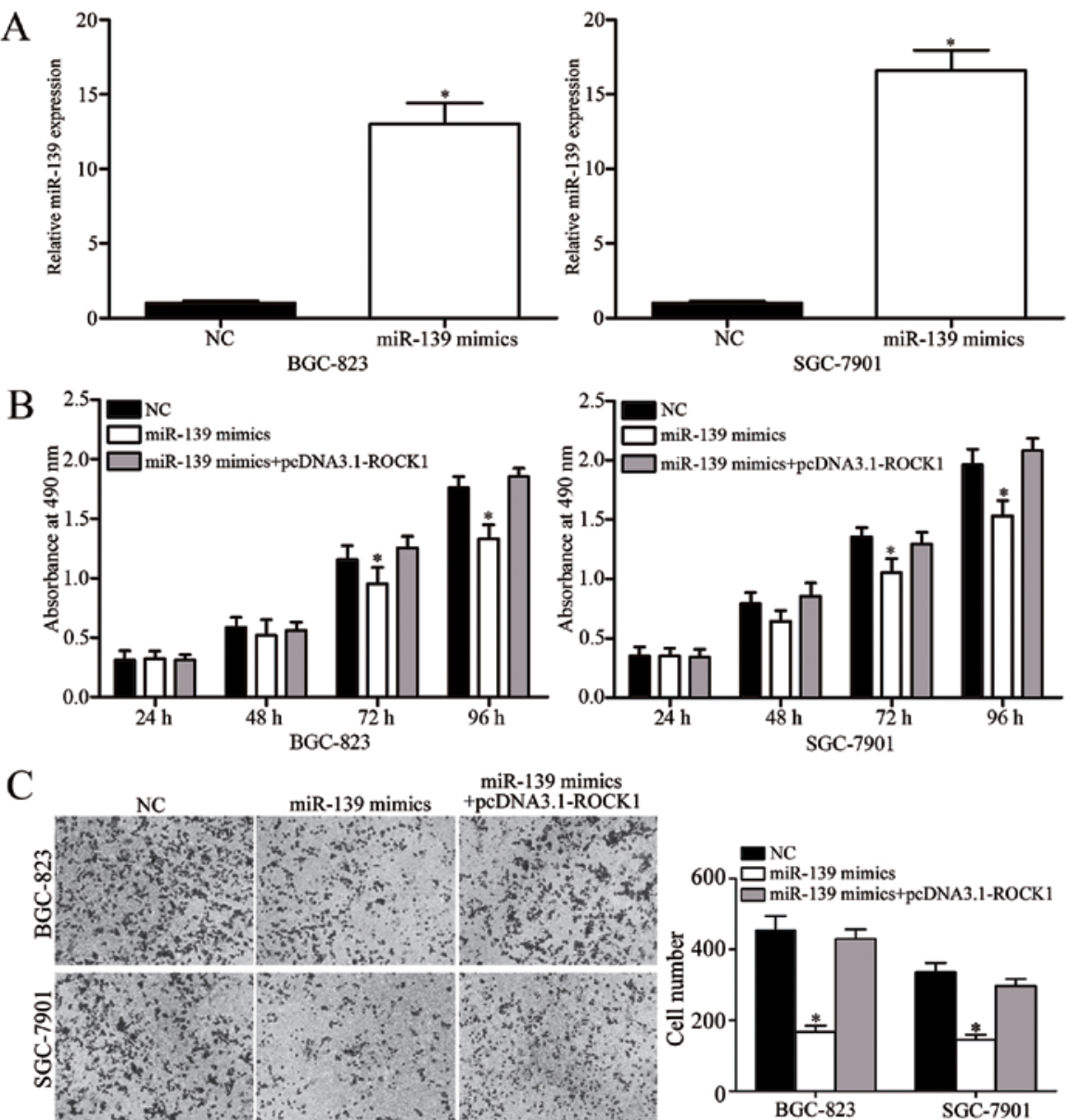

Cell migration assay

$\mathrm{D}$
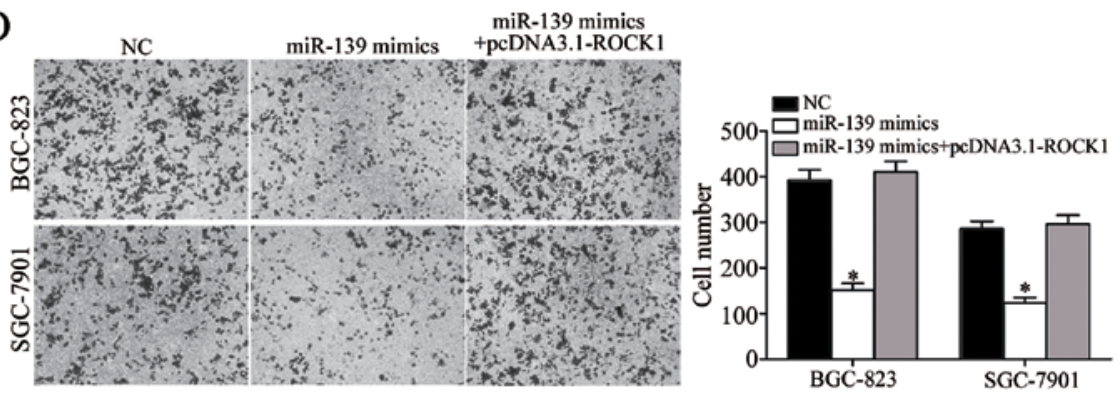

Cell invasion assay
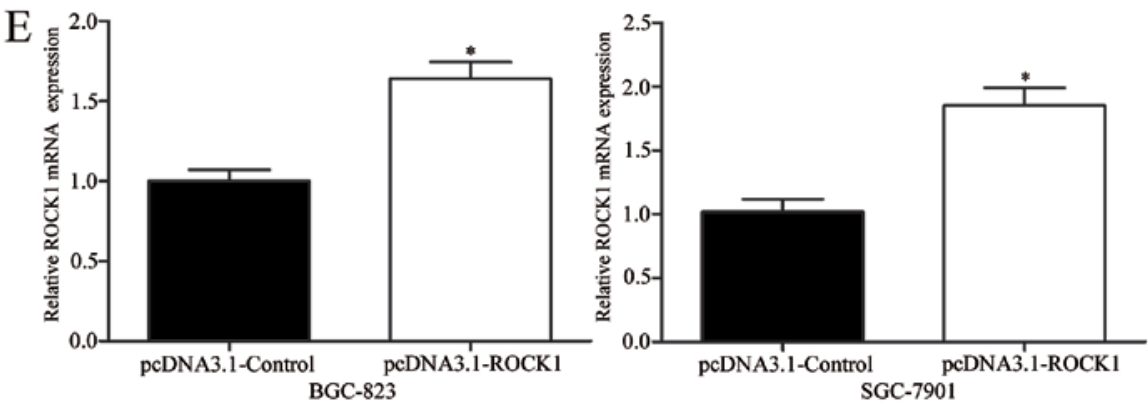

Figure 3. Restored miR-139 expression inhibits gastric cancer cell proliferation, migration and invasion by regulation of ROCK1. (A) miR-139 expression in BGC-823 and SGC-7901 cells transfected with miR-139 mimics or NC was detected at $48 \mathrm{~h}$ post-transfection. (B) The proliferative ability of BGC-823 and SGC-7901 cells co-transfected with NC, miR-139 mimic or miR-139 mimic + pcDNA3.1-ROCK1 was determined. (C) Cell migration and (D) invasion assays of BGC-823 and SGC-7901 cells transfected with NC, miR-139 mimic or miR-139 mimic + pcDNA3.1-ROCK1. (E) ROCK1 mRNA expression in BGC-823 and SGC-7901 cells transfected with pcDNA3.1-ROCK1 or pcDNA3.1-Control. " $\mathrm{P}<0.05$ vs. control. miR-139, microRNA-139; ROCK1, $\rho$-associated protein kinase $1 ; \mathrm{NC}$, negative control.

results demonstrated that miR-139 is a promising target for cancer therapy.
To investigate the molecular mechanisms underlying the cell proliferation, migration and invasion inhibition by 
miR-139, the target genes of miR-139 in gastric cancer were investigated. Bioinformatics analysis with TargetScan and PicTar revealed that the conserved binding sites on the 3'UTR of ROCK1 mRNA can be recognized by miR-139, suggesting that ROCK1 expression may be regulated by miR-139 by binding to the 3'UTR of ROCK. Luciferase reporter assays were performed to validate this hypothesis. Vectors containing the wild-type or the mutant 3'UTR segments of ROCK1 and miR-139 mimic or NC were co-transfected into gastric cancer cells. The luciferase activity of the pGL3-ROCK1-3'UTR-Wtand miR-139 mimic-transfected gastric cells was lower than that of cells transfected with pGL3-ROCK1-3'UTR-Mut and miR-139 mimic, confirming that miR-139 binds to the 3'UTR of ROCK 1 mRNA. The ability of miR-139 to modulate ROCK1 mRNA and protein expression was subsequently investigated. RT-qPCR and western blot analysis revealed that restored miR-139 expression resulted in significantly decreased ROCK1 mRNA and protein expression. Furthermore, ROCK1 overexpression attenuated the effects of miR-139 in gastric cancer cells, indicating that ROCK1 is a functional target of miR-139 in gastric cancer. To the best our knowledge, the present study is the first to investigate miR-139/ROCK1 interactions in gastric cancer.

In conclusion, the downregulation of miR-139 in gastric cancer tissues and cell lines was confirmed, and ectopic miR-139 expression repressed the proliferation, migration and invasion of gastric cancer cells. ROCK1 was validated as a novel direct target gene of miR-139 in gastric cancer. Taken together, the findings of the present study demonstrate that miR-139 downregulation is key in the initiation and progression of gastric cancer, and that miR-139 has therapeutic potential for gastric cancer.

\section{References}

1. Torre LA, Bray F, Siegel RL, Ferlay J, Lortet-Tieulent J and Jemal A: Global cancer statistics, 2012. CA Cancer J Clin 65: 87-108, 2015

2. Zhang YZ, Zhang LH, Gao Y, Li CH, Jia SQ, Liu N, Cheng F, Niu DY, Cho WC, Ji JF and Zeng CQ: Discovery and validation of prognostic markers in gastric cancer by genome-wide expression profiling. World J Gastroenterol 17: 1710-1717, 2011.

3. Tkachenko MA, Zhannat NZ, Erman LV, Blashenkova EL, Isachenko SV, Isachenko OB, Graham DY and Malaty HM: Dramatic changes in the prevalence of Helicobacter pylori infection during childhood: A 10-year follow-up study in Russia J Pediatr Gastroenterol Nutr 45: 428-432, 2007.

4. Wang F, Meng W, Wang B and Qiao L: Helicobacter pylori-induced gastric inflammation and gastric cancer. Cancer Lett 345: 196-202, 2014.

5. Yan C, Yu J, Liu Y, Kang W, Ma Z and Zhou L: MiR-32 promotes gastric carcinoma tumorigenesis by targeting Kruppel-like factor 4. Biochem Biophys Res Commun 467: 913-920, 2015.

6. Kim SJ, Wang YG, Lee HW, Kang HG, La SH, Choi IJ, Irimura T, Ro JY, Bresalier RS and Chun KH: Up-regulation of neogenin-1 increases cell proliferation and motility in gastric cancer. Oncotarget 5: 3386-3398, 2014.

7. Delaunoit T: Latest developments and emerging treatment options in the management of stomach cancer. Cancer Manag Res 3: 257-266, 2011.

8. Bartel DP: MicroRNAs: Genomics, biogenesis, mechanism and function. Cell 116: 281-297, 2004

9. He L and Hannon GJ: MicroRNAs: Small RNAs with a big role in gene regulation. Nat Rev Genet 5: 522-531, 2004.
10. Tahara H, Kay MA, Yasui W and Tahara E: MicroRNAs in Cancer: The 22nd hiroshima cancer seminar/the 4th Japanese Association for RNA interference joint international symposium, 30 August 2012, grand prince hotel hiroshima. Jpn J Clin Oncol 43: 579-582, 2013.

11. Yates LA, Norbury CJ and Gilbert RJ: The long and short of microRNA. Cell 153: 516-519, 2013.

12. Croce CM: Causes and consequences of microRNA dysregulation in cancer. Nat Rev Genet 10: 704-714, 2009.

13. Tang R, Yang C, Ma X, Wang Y, Luo D, Huang C, Xu Z, Liu P and Yang L: MiR-let-7a inhibits cell proliferation, migration and invasion by down-regulating PKM2 in gastric cancer. Oncotarget 7: 5972-5984, 2016.

14. Li J, Dong G, Wang B, Gao W and Yang Q: miR-543 promotes gastric cancer cell proliferation by targeting SIRT1. Biochem Biophys Res Commun 469: 15-21, 2016.

15. Livak KJ and Schmittgen TD: Analysis of relative gene expression data using real-time quantitative PCR and the 2(-Delta Delta C(T)) method. Methods 25: 402-408, 2001.

16. Wang H, Yan X, Ji LY, Ji XT, Wang P, Guo SW and Li SZ: miR-139 functions as an antioncomir to repress glioma progression through targeting IGF-1 R, AMY-1 and PGC-1 $\beta$. Technol Cancer Res Treat 16: 497-511, 2017.

17. Duz MB, Karatas OF, Guzel E, Turgut NF, Yilmaz M, Creighton CJ and Ozen M: Identification of miR-139-5p as a saliva biomarker for tongue squamous cell carcinoma: A pilot study. Cell Oncol (Dordr) 39: 187-193, 2016.

18. Zhang HD, Sun DW, Mao L, Zhang J, Jiang LH, Li J, Wu Y, Ji H, Chen W, Wang J, et al: MiR-139-5p inhibits the biological function of breast cancer cells by targeting Notch1 and mediates chemosensitivity to docetaxel. Biochem Biophys Res Commun 465: 702-713, 2015.

19. Xu W, Hang M, Yuan CY, Wu FL, Chen SB and Xue K: MicroRNA-139-5p inhibits cell proliferation and invasion by targeting insulin-like growth factor 1 receptor in human non-small cell lung cancer. Int J Clin Exp Pathol 8: 3864-3870, 2015.

20. Liu X, Duan B, Dong Y, He C, Zhou H, Sheng H, Gao H and Zhang X: MicroRNA-139-3p indicates a poor prognosis of colon cancer. Int J Clin Exp Pathol 7: 8046-8052, 2014.

21. Wong CC, Wong CM, Tung EK, Au SL, Lee JM, Poon RT, Man K and Ng IO: The microRNA miR-139 suppresses metastasis and progression of hepatocellular carcinoma by down-regulating Rho-kinase 2. Gastroenterology 140: 322-331, 2011.

22. Shen K, Liang Q, Xu K, Cui D, Jiang L, Yin P, Lu Y, Li Q and Liu J.: MiR-139 inhibits invasion and metastasis of colorectal cancer by targeting the type I insulin-like growth factor receptor. Biochem Pharmacol 84: 320-330, 2012.

23. Liu R, Yang M, Meng Y, Liao J, Sheng J, Pu Y, Yin L and Kim SJ: Tumor-suppressive function of miR-139-5p in esophageal squamous cell carcinoma. PLoS One 8: e77068, 2013.

24. Li RY, Chen LC, Zhang HY, Du WZ, Feng Y, Wang HB, Wen JQ, Liu X, Li XF, Sun Y, et al: MiR-139 inhibits Mcl-1 expression and potentiates TMZ-induced apoptosis in glioma. CNS Neurosci Ther 19: 477-483, 2013.

25. Dai S, Wang X, Li X and Cao Y: MicroRNA-139-5p acts as a tumor suppressor by targeting ELTD1 and regulating cell cycle in glioblastoma multiforme. Biochem Biophys Res Commun 467: 204-210, 2015.

26. Yue S, Wang L, Zhang H, Min Y, Lou Y, Sun H, Jiang Y, Zhang W, Liang A, Guo Y, et al: miR-139-5p suppresses cancer cell migration and invasion through targeting ZEB1 and ZEB2 in GBM. Tumour Biol 36: 6741-6749, 2015.

27. Qiu G, Lin Y, Zhang H and Wu D: miR-139-5p inhibits epithelial-mesenchymal transition, migration and invasion of hepatocellular carcinoma cells by targeting ZEB1 and ZEB2. Biochem Biophys Res Commun 463: 315-321, 2015.

28. Zhang L, Dong Y, Zhu N, Tsoi H, Zhao Z, Wu CW, Wang K, Zheng S, Ng SS, Chan FK, et al: microRNA-139-5p exerts tumor suppressor function by targeting NOTCH1 in colorectal cancer. Mol Cancer 13: 124, 2014.

29. Luo HN, Wang ZH, Sheng Y, Zhang Q, Yan J, Hou J, Zhu K, Cheng Y, Xu YL, Zhang XH, et al: MiR-139 targets CXCR4 and inhibits the proliferation and metastasis of laryngeal squamous carcinoma cells. Med Oncol 31: 789, 2014. 